\title{
Spike Protein Fusion Peptide and Feline Coronavirus Virulence
}

\author{
Hui-Wen Chang, Herman F. Egberink, Rebecca Halpin, David J. Spiro, and Peter J.M. Rottier
}

Coronaviruses are well known for their potential to change their host or tissue tropism, resulting in unpredictable new diseases and changes in pathogenicity; severe acute respiratory syndrome and feline coronaviruses, respectively, are the most recognized examples. Feline coronaviruses occur as 2 pathotypes: nonvirulent feline enteric coronaviruses (FECVs), which replicate in intestinal epithelium cells, and lethal feline infectious peritonitis viruses (FIPVs), which replicate in macrophages. Evidence indicates that FIPV originates from FECV by mutation, but consistent distinguishing differences have not been established. We sequenced the full genome of 11 viruses of each pathotype and then focused on the single most distinctive site by additionally sequencing hundreds of viruses in that region. As a result, we identified 2 alternative amino acid differences in the putative fusion peptide of the spike protein that together distinguish FIPV from FECV in $>95 \%$ of cases. By these and perhaps other mutations, the virus apparently acquires its macrophage tropism and spreads systemically.

$\mathrm{C}$ oronaviruses (subfamily Coronavirinae, order Nidovirales) are enveloped, plus-strand RNA viruses that infect mammals and birds. They are quite common and cause infections in humans and a wide variety of animals; infection typically results in respiratory or enteric disease. Severe acute respiratory syndrome coronavirus (SARS$\mathrm{CoV}$ ), which emerged suddenly in 2002 and caused severe acute respiratory disease in humans, is the most notorious coronavirus. SARS-CoV spread rapidly around the globe, infecting thousands and killing $\approx 800$ persons. The virus

Author affiliations: Utrecht University, Utrecht, the Netherlands (H.-W. Chang, H.F. Egberink, P.J.M. Rottier); and J. Craig Venter Institute, Rockville, Maryland, USA (R. Halpin, D.J. Spiro)

DOI: http://dx.doi.org/10.3201/eid1807.120143 presumably originated from bats and was transmitted to humans either directly or by using civets or raccoon dogs as intermediate hosts $(1,2)$.

SARS-CoV best illustrates the remarkable potential for CoVs to change their tropism. Tropism switching has been implicated in the zoonotic emergence of human coronavirus $\mathrm{OC} 43$ from a bovine coronavirus and in turning transmissible gastroenteritis virus, an enteric porcine coronavirus, into porcine respiratory coronavirus, a respiratory pathogen $(3,4)$. Such changes can be accompanied, although not necessarily, by crossspecies transmissions; thus, the erratic occurrence and unpredictable new disease manifestations of tropism switching are a matter of public health concern.

The feline coronaviruses (FCoVs) present an example of pathogenetic change apparently associated with tropism switching. These viruses occur as 2 pathotypes with an enigmatic, even controversial, relationship: the lowvirulence or nonvirulent feline enteric coronavirus (FECV) and the highly lethal feline infectious peritonitis virus (FIPV). FECV and FIPV are considered independently circulating viruses by some investigators $(5,6)$. However, accumulating evidence supports the mutation hypothesis, which proposes that FIPV evolves from FECV by mutation in individually infected cats (7-12). A responsible mutation(s) has not been identified to back this hypothesis.

FECV is ubiquitous and spreads efficiently by the fecal-oral route; hence, seropositivity among cat populations can reach $90 \%$, depending on the field conditions (13). The infection is restricted to the enteric tract, where the virus replicates in epithelial cells lining the gut mucosa. FECV infection is mild, causing transient enteritis that often passes unnoticed. The infection cannot be cleared efficiently by the immune system and thus persists, often for weeks or months, sometimes even longer 
(14-18). In contrast, FIPV is rare, but the consequences of infection are devastating. FIPV infection causes a progressive systemic disease called feline infectious peritonitis. The disease affects many organs, usually inducing fatal immunopathologic disease characterized by disseminated pyogranulomas and severe inflammatory damage to serosal membranes.

In the past, sequence differences in several virus genes, including those encoding membrane and spike (S) structural proteins and the so-called group-specific proteins $3 \mathrm{c}$ and $7 \mathrm{~b}$, have been implicated in the $\mathrm{FCoV}$ virulence shift $(5-7,10,11,19-22)$. However, none of these differences appeared to consistently correlate with disease phenotype. To establish a consistent cause for a virulence shift in FCoV, specifically the predominant serotype I FCoV, we sequenced the entire genome of several FECV and FIPV specimens and then concentrated on the most conspicuous region of consistent difference by collecting and sequencing additional FECV and FIPV samples.

\section{Materials and Methods}

\section{Viruses and Clinical Specimens}

FECV strain RM and FECV strain UCD (FECV UU2) were propagated in specific pathogen-free cats. FIPV UU3 was obtained from a lymph node of a cat infected with FECV UCD; the presence of feline infectious peritonitis in the cat was pathologically confirmed. During 2006-2011, with the assistance of veterinarians in the Netherlands, we randomly obtained field cats with suspected feline infectious peritonitis and feces samples from apparently healthy cats from all geographic areas of the Netherlands; we did not use any selection criteria, such as age, sex, or breed of cat. Cats originated from 144 different catteries and single- and multicat households. Cats were pathologically diagnosed with feline infectious peritonitis by postmortem examination at the Veterinary Pathology Department, Utrecht University; findings confirmed that the cats had feline infectious peritonitis. Ascites samples and lesions from affected organs were obtained for RNA isolation. Fecal material from apparently healthy cats was obtained from the rectum by using a cotton swab.

\section{RNA Preparation}

We suspended fecal specimens to a final concentration of $10 \%$ (wt/vol) in phosphate-buffered saline by vigorously vortexing the specimens. The supernatant was cleared (centrifugation for $10 \mathrm{~min}$ at $3,000 \times \mathrm{g}$ ) and then used for RNA extraction. Following the manufacturer's protocols, we used the QIAamp Viral RNA Mini Kit (QIAGEN, Valencia, CA, USA) to extract viral RNA from $140 \mu \mathrm{L}$ of fecal supernatants or ascites and the QIAamp RNeasy Mini Kit to extract viral RNA from $30 \mathrm{mg}$ of organ tissue homogenate.

\section{FCoV Detection and Serotyping}

We tested RNA isolated from organs or ascites of cats with feline infectious peritonitis and from feces of apparently healthy cats for the presence of FCoV RNA by using a reverse transcription nested PCR (RT-nPCR) targeting the highly conserved $3 \times$ untranslated region (23). Samples with results positive for FCoV were checked for the virus serotype by using an RT-nPCR targeting the S gene (24). Only samples positive for serotype I FCoV were included in this study.

We determined the sequence in the $\mathrm{S}$ gene region of interest by using an RT-nPCR. In brief, we synthesized complementary DNA by using an antisense primer (5'-CCCTCGAGTCCCGCAGAAACCATACCTA-3') and superscript II reverse transcriptase (Promega, Madison, WI, USA) at $50^{\circ} \mathrm{C}$ for $1 \mathrm{~h}$. We then performed the PCR by using Taq DNA polymerase (Promega) and specific primers (sense 5'-CAATATTACAATGGCATAATGG-3', antisense 5'-CC CTCGAGTCCCGCAGAAACCATACCTA-3') for the first reaction and specific primers (sense 5'-GGCATAATG GTTTTACCTGGTG-3', antisense 5'-TAATTAAGCCT CGCCTGCACTT-3') for the second reaction. PCR cycling conditions were 30 cycles at $94^{\circ} \mathrm{C}$ for $60 \mathrm{~s}$, at $50^{\circ} \mathrm{C}$ for $30 \mathrm{~s}$, and at $72^{\circ} \mathrm{C}$ for $1 \mathrm{~min}$ plus a $7-$ min extension at $72^{\circ} \mathrm{C}$ at the end of the reaction. All enzymes were used according to the manufacturer's instructions. Primer pairs were expected to generate a 598-bp product for the first PCR run and a 142bp product for the second run.

Representative PCR products were purified by electrophoresis in 2\% agarose gel followed by extraction from the gel by using a gel extraction kit (QIAGEN) according to the manufacturer's recommended instructions. Macrogen Inc. (http://dna.macrogen.com/eng/) sequenced the gel-purified DNA.

\section{Full Genome Sequencing}

Two 96-well plates of degenerate primers (online Technical Appendix, www.cdc.gov/eid-static/spreadsheets/ 12-0143-Techapp.xls) were designed from aligned reference genomes by using a computational PCR primer design pipeline. The pipeline was developed at the J. Craig Venter Institute (JCVI) to produce tiled amplicons with an optimal length of $550 \mathrm{bp}$, with 100-bp overlap to provide 6 -fold sequence coverage of the genome. An M13 sequence tag was added to the $5 \times$ end of each degenerate primer and was used for sequencing. Primers were arranged in a 96-well plate format, and all PCRs for each sample were performed in 2 plates. The primers used in this study are listed in the online Technical Appendix.

Sequencing reactions were performed by using Big Dye Terminator (Applied Biosystems, Foster City, CA, USA) chemistry. Each amplicon was sequenced from both ends by using M13 primers, and sequencing reactions 
were analyzed by using a $3730 \mathrm{ABI}$ sequencer (Applied Biosystems). Raw sequence data were trimmed to remove any primer-derived and low-quality sequence; gene sequences were assembled by using a viral assembly tool (www.jcvi.org/cms/research/software). Assemblies were edited computationally and manually. When insufficient underlying sequence information was obtained, the sample was entered into the secondary sequencing pipeline and reamplified by using existing primers or primers designed from the problematic sequence assembly itself. The reamplified sample was then sequenced again.

An RNA virus genome prediction program called VIGOR (Viral Genome ORF Reader, JCVI (www.jcvi. org/vigor) can decode many classes of viruses, taking into account virus-specific features, such as alternative splicing, internal open reading frames, and ribosomal slippage. This program was used to annotate de novo assemblies of coronaviruses sequenced at JCVI and also to validate newly assembled genomes during the finishing process. Last, we performed a quality control assessment and manually inspected the gene predictions before loading them into the annotation database at JCVI, from which they were exported in formats acceptable to the National Center for Biotechnology Information.

\section{Multiple-Sequence Alignment}

The full-length and partial FCoV genomic nucleotide sequences we obtained were deposited in the National Center for Biotechnology Information database (www.ncbi. nlm.nih.gov). The sequence accession numbers of the fulllength $\mathrm{FCoV}$ sequences used in this study are listed in Table 1. The GenBank accession numbers for the partial S gene sequences are JQ304323-JQ304518. Multiple-sequence alignments were constructed by using Clustal W (www.ebi. ac.uk/clustalw) with the Lasergene MegAlign (DNASTAR, www.dnastar.com/t-sub-products-lasergene-megalign. aspx) and MEGA4 (www.megasoftware.net) software programs. To identify key differences between FIPV and FECV, we analyzed their genomes and proteomes; for each nucleotide or amino acid position, we determined the rate at which FIPVs differed from all FECVs at that position. Phylogenetic analysis was performed by using features of the MEGA4 suite of programs. Phylogenetic trees of these sequences were obtained by using the neighbor-joining method. The bootstrap consensus tree, inferred from 1,000 replicates, was prepared; positions containing gaps and missing data were eliminated from the dataset.

\section{Results}

\section{Full Genome Sequencing}

To identify the distinguishing difference(s) between the FCoV pathotypes, we initiated a full genome sequencing
Table 1. GenBank accession numbers for viruses for which the genomes were fully sequenced in a study to distinguish virulent from nonvirulent feline coronaviruses

\begin{tabular}{lc}
\hline Virus strain & Accession no. \\
\hline Feline infectious peritonitis viruses & \\
UU3 & FJ938061 \\
UU4 & FJ938054 \\
UU5 & FJ938056 \\
UU8 & FJ938055 \\
UU9 & FJ938062 \\
UU15 & FJ938057 \\
UU16 & FJ938058 \\
UU17 & HQ012367 \\
UU21 & HQ012369 \\
UU24 & HQ012370 \\
UU30 & HQ392472 \\
\hline Feline enteric coronaviruses & \\
RM & FJ938051 \\
UU2 (UCD) & FJ938060 \\
UU7 & FJ938053 \\
UU10 & FJ938059 \\
UU11 & FJ938052 \\
UU18 & HQ012368 \\
UU19 & HQ392490 \\
UU20 & HQ392471 \\
UU22 & GU553361 \\
UU23 & GU553362 \\
UU31 & HQ012371 \\
\hline
\end{tabular}

program of FECVs found in the feces of apparently healthy cats and of FIPVs found in organs or ascites of cats with pathologically confirmed feline infectious peritonitis. To obtain a more extensive analysis of the coronavirus genome, this sequencing program is still ongoing; however, after the sequences of 11 genomes of each pathotype were completed, we performed a comparative FECV-FIPV analysis, screening the genomes for nucleotide differences (Table 1). This was done by counting, for every nucleotide position, the number of FIPV genomes for which the identity at that position differed from that in all FECV genomes.

Our results showed that differences were scattered along the entire genome (Figure 1). At 2,963 (10\%) of the 29,277 genome positions, the nucleotide identity in at least 1 of the 11 FIPVs did not occur in any of the 11 FECVs. Of these 2,963 positions, 1,187 occurred in gene 1ab, 1,246 in the S gene, 248 in gene cluster 3abc, 22 in the envelope protein gene, 42 in the membrane protein gene, 113 in the nucleocapsid protein gene, and 106 in gene cluster 7ab, showing the disproportionally large genetic variation in the $\mathrm{S}$ gene. The frequency with which differences occurred at different nucleotide positions across the genome showed the following distribution: a difference was detected $1 \times$ at 1,914 positions, $2 \times$ at 945 positions, $3 \times$ at 87 positions, $4 \times$ at 15 positions, and $5 \times$ at 1 position. At 1 position (23531), the nucleotide identity in 9 of the FIPVs was not found in any of the FECVs. No position(s) uniquely distinguished the $2 \mathrm{FCoV}$ pathotypes.

Nucleotide identity differed the most at position 23531: it was highly conserved $(100 \% \mathrm{~A})$ in all FECV 


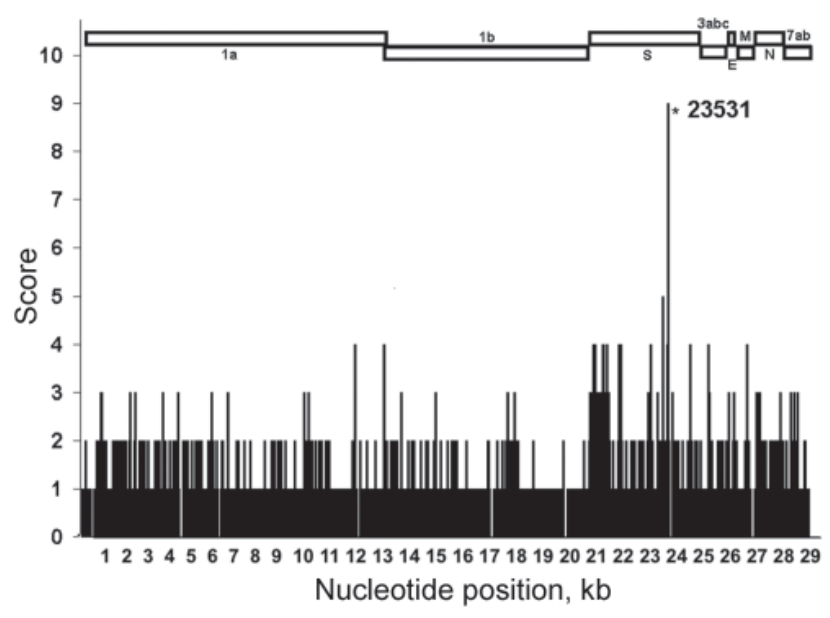

Figure 1. Comparison of full genomes of 11 lethal feline infectious peritonitis viruses (FIPVs) with full genomes of 11 nonvirulent feline enteric coronaviruses (FECVs). Nucleotide (nt) positions are shown on the x-axis; $y$-axis indicates number of FIPV genomes for which the identity at the nt position differed from identity at same position in all FECV genomes. FIPV strain C1Je (GenBank accession no. DQ848678) was used as the reference for nt numbering. *Highest difference score: 9 FIPVs had identities at nt position 23531 that differed from those at the same position in all FECVs. 1a, gene $1 \mathrm{a} ; 1 \mathrm{~b}$, gene $1 \mathrm{~b} ; \mathrm{S}$, spike protein gene; 3abc, gene cluster 3abc; E, envelope protein gene; $M$, membrane protein gene; $N$, nucleocapsid protein gene; $7 \mathrm{ab}$, gene cluster $7 \mathrm{ab}$.

genomes, and it was $\mathrm{C}$ or $\mathrm{T}$ in 9 of the 11 FIPV genomes. This difference occurs in the $\mathrm{S}$ gene and results in an amino acid difference in the predicted $\mathrm{S}$ protein. Thus, although all FECV S proteins have a methionine at position 1058, a leucine is encoded in the 9 FIPVs, irrespective of the identity of the genetic difference (C or T).

\section{Sequencing of the $\mathrm{S}$ gene}

To further investigate the single most prominent region of difference between FECVs and FIPVs, we established an RT-nPCR method to amplify and analyze the genomic region covering nucleotides 23442-24040 for the first PCR run and nucleotides 23451-23593 for the second run, which includes deviant position 23531. Altogether, $183 \mathrm{FECV}$ and 118 FIPV RNAs isolated from different cats were sequenced in this specific region. Results for the 11 entirely sequenced FECVs and FIPVs are shown in Figure 2. The A at nucleotide 23531 was $100 \%$ conserved in all 183 FECVs in our collection. Of the 118 FIPVs, $96(81.4 \%)$ had a $\mathrm{T}$ and $12(10.2 \%)$ a $\mathrm{C}$ at this position; in both cases, this changes the methionine occurring at position 1058 in the FECV S protein into a leucine in FIPV (i.e., mutation M1058L).

\section{Phylogenetic Analysis}

Assuming that the difference observed in 108 of the 118 sequenced FIPVs may be responsible for the virulent phenotype, the remaining 10 viruses should be expected to carry alternative differences. In search of those differences, we performed a phylogenetic analysis of the partial nucleotide sequences accumulated by the RT-nPCR procedure, but the results did not enable further differentiation (data not shown). When carrying out a phylogenetic analysis of the translated partial amino acid sequences, we observed a small but distinct second cluster $\mathrm{B}$ in addition to the major cluster A constituted by the FIPVs having leucine at position 1058 in their S protein (Figure 3). This smaller cluster, formed by $5(4.2 \%)$ of the sequenced FIPVs, appeared to be characterized by the occurrence of an alanine at position 1060 (i.e., mutation S1060A), just 2 residues downstream of the $\mathrm{M}^{1058}$ that is changed in most FIPVs. All other FIPVs and all sequenced FECVs consistently had a serine at this position. The difference is brought about in all 5 cases by a $\mathrm{T} \rightarrow \mathrm{G}$ change at nucleotide 23537 . Overall, we have detected characteristic differences with FECV for 113 (95.8\%) of the 118 sequenced FIPVs. These differences were observed in both pathologic forms (i.e., wet and dry forms) of feline infectious peritonitis (Table 2).

\section{Discussion}

Our findings show differences in 2 alternative codons of the FCoV S gene that correlate with the feline infectious peritonitis disease phenotype in $>95 \%$ of cases. Besides providing a realistic basis for diagnostic discrimination of the $2 \mathrm{FCoV}$ pathotypes, our findings also support the

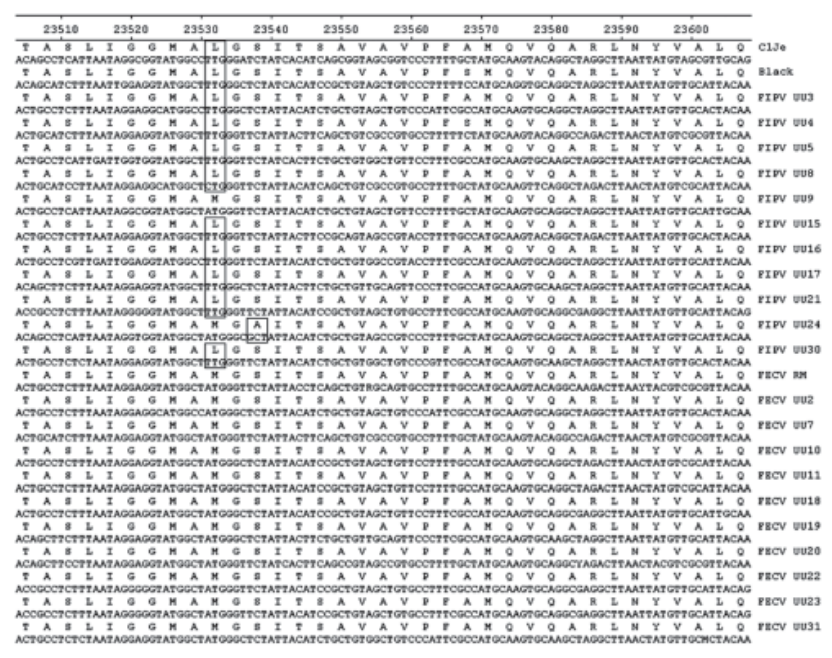

Figure 2. Alignment of partial nucleotide sequences and translated amino acid sequences in the spike protein of 11 strains each of 2 feline coronavirus pathotypes: FIPVs (lethal) and FECVs (nonvirulent). The viruses were sequenced in a study to distinguish virulent from nonvirulent feline coronaviruses (see Table 1). FIPV strain C1Je (GenBank accession no. DQ848678) was used as the reference for numbering. Sequence positions are shown along the top; virus strains are shown on the right. Specific differences between the pathotypes are boxed. FIPVs, feline infectious peritonitis viruses; FECVs, feline enteric coronaviruses. 


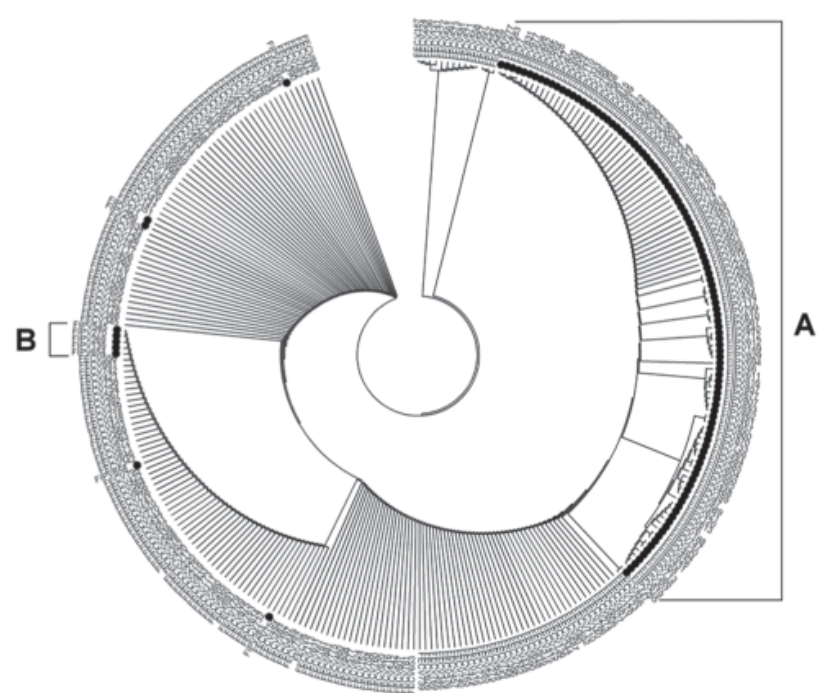

Figure 3. Phylogenetic tree based on partial amino acid sequences (aa 1056-1069) of the spike proteins of 118 feline infectious peritonitis viruses (FIPVs) and 183 feline enteric coronaviruses (FECVs) obtained by using reverse transcription nested PCR and sequencing of the distinguishing genomic region. A circular rooted neighbor-joining tree was constructed by using the bootstrap method and applying 1,000 replicates. Black dots indicate FIPVs. Clade A comprises FIPVs containing the M1058L mutation; clade B comprises FIPVs containing the S1060A mutation.

mutation hypothesis. Thus, we propose that alternative mutations in the S protein of FECV give rise to a tropism change that allows the virus to escape from the intestine into body tissues, where it causes feline infectious peritonitis. Proof of this hypothesis will require introduction of these mutations into the FECV genome and demonstration of the virulence switch by infection of cats. However, this is a formidable challenge in the absence of a reverse genetics system and a proper cell culture system to generate and propagate these viruses.

Our findings relating the $\mathrm{S}$ protein to $\mathrm{FCoV}$ pathogenicity are not surprising, given earlier explorations into the involvement of various genes $(7 \mathrm{a}, 7 \mathrm{~b}, \mathrm{M}$, and 3c) $(5-7,10,11,19-22,24,25)$. One of the most notable consequences of the presumed mutation in FECV is the acquisition of monocyte/macrophage tropism by the resulting virus (26). Thus, whereas replication of FECV is restricted to the epithelial cells lining the gut, the virulence mutation enables FIPV to efficiently infect and replicate in macrophages and spread the infection systemically (26). Such tropism change corresponds most logically with a modification in the $\mathrm{S}$ protein. An earlier study, using serotype II FCoVs, indicated a virulence role for the S protein (21); however, identification of the mutation(s) was not pursued because of the controversial nature of the FECV strain used in the study (14).
As for the serotype II viruses, the putative virulence mutations detected in the serotype I FCoV spike occur in the membrane-proximal domain of the protein. In coronaviruses, the $\mathrm{S}$ protein functions in cell entry; it is responsible for receptor attachment and membrane fusion. While the receptor binding site is located in the $\mathrm{N}$ terminal part of the protein, fusion is mediated by its membraneproximal part. Coronavirus S proteins are class I fusion proteins, which typically contain domains instrumental for this process: 2 heptad repeat regions and a fusion peptide (27). The fusion peptide is located just upstream of the membrane-distal heptad repeat region, but it remains to be proven that it functions as a fusion peptide. The 2 putative virulence mutations identified in our study, M1058L and S1060A, map to this characteristic hydrophobic domain. Both changes are subtle and do not give clues as to their functional consequences. We assume, however, that these alternative mutations have a similar effect, and we speculate that the mutations in the remaining $4 \%$ of cases might also occur in the fusion peptide of the S protein.

If these mutations are all that is needed to convert a nonvirulent FECV into a lethal FIPV, the question arises as to why feline infectious peritonitis occurs so infrequently. For example, simple calculations based on a $10^{-4}$ frequency and a stochastic occurrence of RNA polymerase errors across the genome (28) predict that the M1058L mutation, for which 2 alternative substitutions of $\mathrm{A}^{23531}$ (to $\mathrm{T}$ or $\mathrm{G}$ ) occur, would statistically arise once in every $1.5 \times 10^{4}$ genomes produced. In experimental FECV infection of kittens, we showed that up to $10^{8}$ genome equivalents of the virus are shed per microliter of feces (18); thus, typical FECV infections would be expected to generate thousands of progeny carrying 1 of the critical mutations. However, the virulence phenotype supposedly associated with the mutation is not observed to any proportional extent. We can only speculate as to the reasons.

One likely possibility is that additional mutations (1 or more, perhaps alternative mutations) are required to generate the virulent pathotype. Such mutations would most probably involve the accessory gene $3 \mathrm{c}$, which is intact in FECVs but severely affected in about two thirds of FIPVs $(7,10-12)$. The $3 \mathrm{c}$ protein apparently is

\begin{tabular}{lcc}
\hline \multicolumn{3}{l}{ Table 2. Prevalence of alternative mutations in feline infectious } \\
peritonitis virus spike protein of cats with wet and dry forms of \\
FIP* \\
\hline \\
\cline { 2 - 3 } Mutation & Pathologic form of FIP \\
\hline M1058L & 71 & Dry \\
S1060A & 5 & 46 \\
No mutation & 3 & 1 \\
\hline Total & 79 & 3 \\
\hline${ }^{*}$ Cats were diagnosed with the dry or wet form of FIP (feline infectious \\
peritonitis) during postmortem examination by a veterinary pathologist. \\
The occurrence and nature of the mutation in the spike gene was \\
established by sequencing.
\end{tabular}


essential for replication of FECV in the gut but becomes nonessential once virulence mutation(s) elsewhere in the genome (e.g., in the $\mathrm{S}$ gene) enable the virus to infect macrophages and spread systemically. As we suggested earlier, loss of $3 \mathrm{c}$ function may not only be tolerated, it may even enhance the fitness of the mutant virus in its new biotope and, as a consequence, hamper its return to the gut. If the mutant virus is absent in the gut, it will not be shed in feces, providing an explanation for the seemingly rare incidence of feline infectious peritonitis outbreaks. Our discoveries of the critical differences between FECVs and FIPVs are clearly only a small step toward understanding the pathogenetic phenomena of feline coronavirus infections.

\section{Acknowledgment}

We thank Niels Pedersen for providing FECV strain RM and FECV strain UCD.

This project was funded in part by the National Institute of Allergy and Infectious Diseases, National Institutes of Health, Department of Health and Human Services (contract no. HHSN272200900007C).

Dr Chang is a veterinary postdoctoral researcher in the Virology Division, Department of Infectious Diseases and Immunology, Veterinary Faculty, Utrecht University, the Netherlands. Her research interests include viral pathogenesis and veterinary virology, pathology, and immunology.

\section{References}

1. Perlman S, Netland J. Coronaviruses post-SARS: update on replication and pathogenesis. Nat Rev Microbiol. 2009;7:439-50. http:// dx.doi.org/10.1038/nrmicro2147

2. Weiss SR, Leibowitz JL. Coronavirus pathogenesis. Adv Virus Res. 2011;81:85-164.

3. Laude H, Van Reeth K, Pensaert M. Porcine respiratory coronavirus: molecular features and virus-host interactions. Vet Res. 1993;24:125-50.

4. Vaughn EM, Halbur PG, Paul PS. Sequence comparison of porcine respiratory coronavirus isolates reveals heterogeneity in the $\mathrm{S}, 3$, and 3-1 genes. J Virol. 1995;69:3176-84.

5. Brown MA, Troyer JL, Pecon-Slattery J, Roelke ME, O'Brien SJ. Genetics and pathogenesis of feline infectious peritonitis virus. Emerg Infect Dis. 2009;15:1445-52. http://dx.doi.org/10.3201/ eid1509.081573

6. Brown MA. Genetic determinants of pathogenesis by feline infectious peritonitis virus. Vet Immunol Immunopathol. 2011;143:2658. http://dx.doi.org/10.1016/j.vetimm.2011.06.021

7. Chang HW, de Groot RJ, Egberink HF, Rottier PJ. Feline infectious peritonitis: insights into feline coronavirus pathobiogenesis and epidemiology based on genetic analysis of the viral 3c gene. J Gen Virol. 2010;91:415-20. http://dx.doi.org/10.1099/vir.0.016485-0

8. Chang HW, Egberink HF, Rottier PJ. Sequence analysis of feline coronaviruses and the circulating virulent/avirulent theory. Emerg Infect Dis. 2011;17:744-6.
9. Poland AM, Vennema H, Foley JE, Pedersen NC. Two related strains of feline infectious peritonitis virus isolated from immunocompromised cats infected with a feline enteric coronavirus. J Clin Microbiol. 1996;34:3180-4.

10. Vennema H, Poland A, Foley J, Pedersen NC. Feline infectious peritonitis viruses arise by mutation from endemic feline enteric coronaviruses. Virology. 1998;243:150-7. http://dx.doi.org/10.1006/ viro.1998.9045

11. Pedersen NC, Liu H, Dodd KA, Pesavento PA. Significance of coronavirus mutants in diseased tissues of cats suffering from feline infectious peritonitis. Viruses. 2009;1:166-84. http://dx.doi. org/10.3390/v1020166

12. Pedersen NC, Liu H, Scarlett J, Leutenegger CM, Golovko L, Kennedy H, et al. Feline infectious peritonitis: role of the feline coronavirus $3 \mathrm{c}$ gene in intestinal tropism and pathogenicity based upon isolates from resident and adopted shelter cats. Virus Res. 2012;165:17-28. http://dx.doi.org/10.1016/j.virusres.2011.12.020

13. Pedersen NC, Sato R, Foley JE, Poland AM. Common virus infections in cats, before and after being placed in shelters, with emphasis on feline enteric coronavirus. J Feline Med Surg. 2004;6:83-8. http://dx.doi.org/10.1016/j.jfms.2003.08.008

14. Pedersen NC, Allen CE, Lyons LA. Pathogenesis of feline enteric coronavirus infection. J Feline Med Surg. 2008;10:529-41. http:// dx.doi.org/10.1016/j.jfms.2008.02.006

15. Pedersen NC. A review of feline infectious peritonitis virus infection: 1963-2008. J Feline Med Surg. 2009;11:225-58. http://dx.doi. org/10.1016/j.jfms.2008.09.008

16. Kipar A, Meli ML, Baptiste KE, Bowker LJ, Lutz H. Sites of feline coronavirus persistence in healthy cats. J Gen Virol. 2010;91:1698707. http://dx.doi.org/10.1099/vir.0.020214-0

17. Herrewegh AA, Mahler M, Hedrich HJ, Haagmans BL, Egberink HF, Horzinek MC, et al. Persistence and evolution of feline coronavirus in a closed cat-breeding colony. Virology. 1997;234:349-63. http://dx.doi.org/10.1006/viro.1997.8663

18. Vogel L, Van der Lubben M, te Lintelo EG, Bekker CP, Geerts T, Schuijff LS, et al. Pathogenic characteristics of persistent feline enteric coronavirus infection in cats. Vet Res. 2010;41:71. http:// dx.doi.org/10.1051/vetres/2010043

19. Sharif S, Arshad SS, Hair-Bejo M, Omar AR, Zeenathul NA, Alazawy A. Diagnostic methods for feline coronavirus: a review. Vet Med Int. 2010:2010:pii:809480.

20. Herrewegh AA, Vennema H, Horzinek MC, Rottier PJ, de Groot RJ. The molecular genetics of feline coronaviruses: comparative sequence analysis of the ORF7a/7b transcription unit of different biotypes. Virology. 1995;212:622-31. http://dx.doi.org/10.1006/ viro. 1995.1520

21. Rottier PJ, Nakamura K, Schellen P, Volders H, Haijema BJ. Acquisition of macrophage tropism during the pathogenesis of feline infectious peritonitis is determined by mutations in the feline coronavirus spike protein. J Virol. 2005;79:14122-30. http://dx.doi. org/10.1128/JVI.79.22.14122-14130.2005

22. Takano T, Tomiyama Y, Katoh Y, Nakamura M, Satoh R, Hohdatsu T. Mutation of neutralizing/antibody-dependent enhancing epitope on spike protein and $7 \mathrm{~b}$ gene of feline infectious peritonitis virus: influences of viral replication in monocytes/macrophages and virulence in cats. Virus Res. 2011;156:72-80. http://dx.doi.org/10.1016/j. virusres.2010.12.020

23. Herrewegh AA, de Groot RJ, Cepica A, Egberink HF, Horzinek MC, Rottier PJ. Detection of feline coronavirus RNA in feces, tissues, and body fluids of naturally infected cats by reverse transcriptase PCR. J Clin Microbiol. 1995;33:684-9.

24. Addie DD, Schaap IA, Nicolson L, Jarrett O. Persistence and transmission of natural type I feline coronavirus infection. J Gen Virol. 2003;84:2735-44. http://dx.doi.org/10.1099/vir.0.19129-0 
25. Myrrha LW, Silva FMF, de Oliveira Peternelli EF, Junior AS, Resende M, de Almeida MR. The paradox of feline coronavirus pathogenesis: a review. Adv Virol. 2011;2011:109849. http://dx.doi. org/10.1155/2011/109849

26. Stoddart CA, Scott FW. Intrinsic resistance of feline peritoneal macrophages to coronavirus infection correlates with in vivo virulence. J Virol. 1989;63:436-40.

27. Bosch BJ, van der Zee R, de Haan CA, Rottier PJ. The coronavirus spike protein is a class I virus fusion protein: structural and functional characterization of the fusion core complex. J Virol. 2003;77:880111. http://dx.doi.org/10.1128/JVI.77.16.8801-8811.2003
28. Fu K, Baric RS. Map locations of mouse hepatitis virus temperaturesensitive mutants: confirmation of variable rates of recombination. J Virol. 1994;68:7458-66.

Address for correspondence: Peter J.M. Rottier, Virology Division, Department of Infectious Diseases and Immunology, Veterinary Faculty, Utrecht University, Yalelaan 1, 3584 CL Utrecht, the Netherlands; email: p.rottier@uu.nl

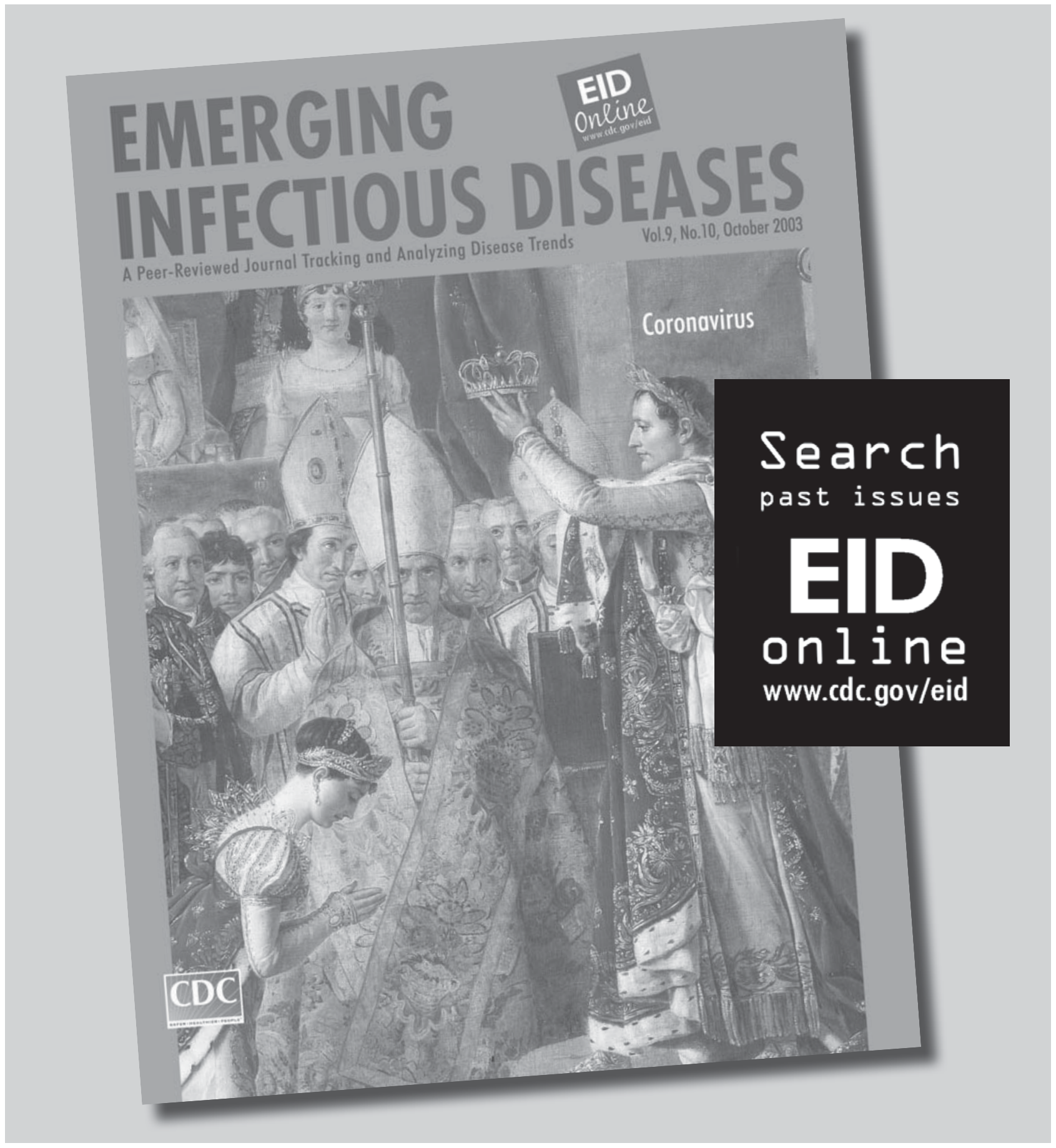

